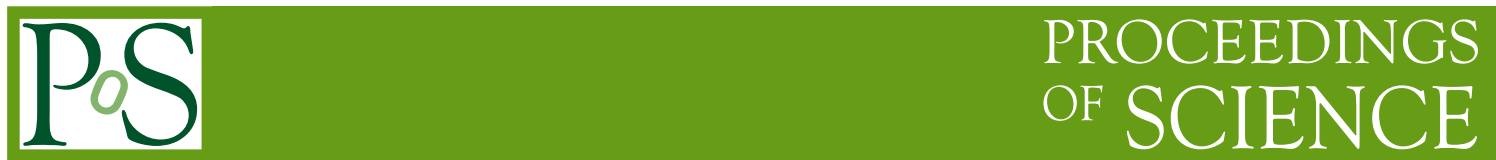

\title{
TMD Evolution: the Small-x Perspective
}

\author{
Yuri V. Kovchegov** \\ Department of Physics, The Ohio State University, Columbus, OH 43210, USA \\ E-mail: kovchegov.1@osu.edu

\section{Matthew D. Sievert} \\ Bldg. 510A, Physics Department, Brookhaven National Laboratory, Upton, NY 11973, USA \\ E-mail: msievert@bnl.gov
}

One aim of this work is to reproduce the leading-order CSS evolution equation for transversemomentum-dependent parton distribution functions (TMDs) using the recently-developed methods of non-linear small- $x$ evolution. The other aim is to use the small- $x$ evolution methods to develop new evolution equations for the small- $x$ TMDs. This presentation is based on [1], with the first half of that paper dedicated to calculating TMDs in the quasi-classical approximation presented in a seperate talk by Matthew Sievert (these proceedings).

QCD Evolution 2015,

26-30 May 2015

Jefferson Lab (JLAB), Newport News Virginia, USA

* Speaker.

${ }^{\dagger}$ This material is based upon work supported by the U.S. Department of Energy, Office of Science, Office of Nuclear Physics under Award Number DE-SC0004286. MS is supported under DOE Contract No. DE-SC0012704. 


\section{Large- $x$ Evolution}

In this Section we deal with QCD evolution at large values of Bjorken $x$ variable. More precisely we consider semi-inclusive deep inelastic scattering (SIDIS) process with $s \sim Q^{2} \gg \perp^{2}$, where $s$ is the center of mass energy of the virtual photon-target system, $Q^{2}$ is the photon virtuality, and $\perp$ denotes the typical transverse momentum in the process. It is natural to assume that since $x=Q^{2} /\left(s+Q^{2}\right)$ is order-one, the small- $x$ evolution methods are not applicable, since we are in a different kinematic regime. However, such conclusion does not take into consideration the fact that there is an effective very small Bjorken- $x$ in the problem, $x_{e f f}=\perp^{2} / s \ll 1$, which, in fact, justifies the use of small- $x$ evolution methods in the calculation.

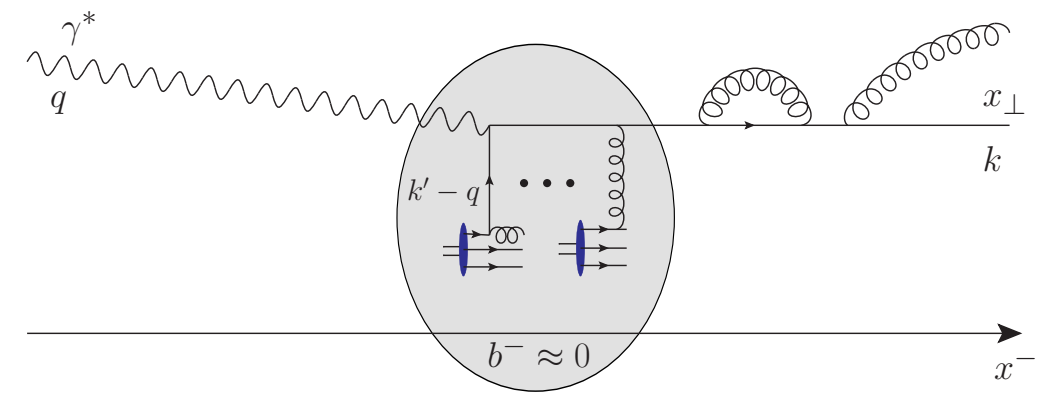

Figure 1: An example of quantum evolution corrections to the SIDIS process and for corresponding quark TMDs.

Let us concentrate on evolution correction summing leading logarithms of energy, that is powers of $\alpha_{s} \ln \left(s / k_{T}^{2}\right)$. Since $s \approx Q^{2}$, we have $\alpha_{s} \ln \left(s / k_{T}^{2}\right) \approx \alpha_{s} \ln \left(Q^{2} / k_{T}^{2}\right)$, and our evolution in the end will become a $Q^{2}$-evolution. Note that the resummation parameter will actually turn out to be double-logarithmic (DLA), that is we will be resumming powers of $\alpha_{s} \ln ^{2}\left(Q^{2} / k_{T}^{2}\right)$. To construct this evolution equation we can use the established formalism of saturation physics $[2,3,4,5,6,7,8,9,10,11,12]$. For definitiveness, we will consider quark TMDs.

We work in the frame where the incoming nucleus has a large $P^{+}$momentum, while the incoming virtual photon has a large positive $q^{-}$along with a comparable but negative $q^{+}$momentum. (While for definitiveness we consider the SIDIS process, all our discussion and conclusions apply to Drell-Yan (DY) process as well.) We will work in the $A^{-}=0$ light-cone gauge.

The typical quantum evolution corrections to a SIDIS amplitude are shown in Fig. 1 (see the real and virtual gluons attached to the outgoing quark line). Our choice of $A^{-}=0$ gauge prevents gluons from being emitted or absorbed by the partons in the incoming nucleons or by the struck quark (carrying momentum $k^{\prime}-q$ in Fig. 1); all these particles move predominantly in the $P^{+}$direction and do not emit gluons in the $A^{-}=0$ gauge in the leading-logarithmic approximation. Therefore, gluon emission and absorption is limited to the outgoing quark. Additionally, logarithms of energy can be generated (in $A^{-}=0$ gauge) only by emissions which happen over long periods of time; therefore, gluon emissions inside the nucleus do not lead to logarithms of energy. We are left only with the gluon emissions and absorptions by the outgoing quark after it exits the nucleus, as shown in Fig. 1.

Such emissions are easy to sum up. Using crossing symmetry, the correction to a light-cone Wilson line at $x_{\perp}$ in the amplitude and another light-cone Wilson line at $y_{\perp}$ in the complex conju- 
gate amplitude, as appears in the cross section, can be accounted for by calculating corrections to a pair of Wilson lines in the amplitude: one at $x_{\perp}$ another one at $y_{\perp}$. This is illustrated in Fig. 2, where evolution corrections (real and virtual gluons) are limited only to corrections for a pair of semi-infinite light-cone Wilson lines, which together form a semi-infinite quark dipole operator

$$
S_{x y}\left[\infty^{-}, b^{-}\right] \equiv \frac{1}{N_{c}}\left\langle\operatorname{Tr}\left[V_{x}\left[\infty^{-}, b^{-}\right] V_{y}^{\dagger}\left[\infty^{-}, b^{-}\right]\right]\right\rangle,
$$

where

$$
V_{x}\left[\infty^{-}, b^{-}\right] \equiv \mathscr{P} \exp \left[i g \int_{b^{-}}^{\infty} d z^{-} g_{+-} A^{+a}\left(0^{+}, z^{-}, \underline{x}\right) t^{a}\right]
$$

is a Wilson line in the fundamental representation and $t^{a}$ is the fundamental generator of $\mathrm{SU}\left(N_{c}\right)$. The angle brackets in Eq. (1.1) denote averaging in the nuclear wave function.

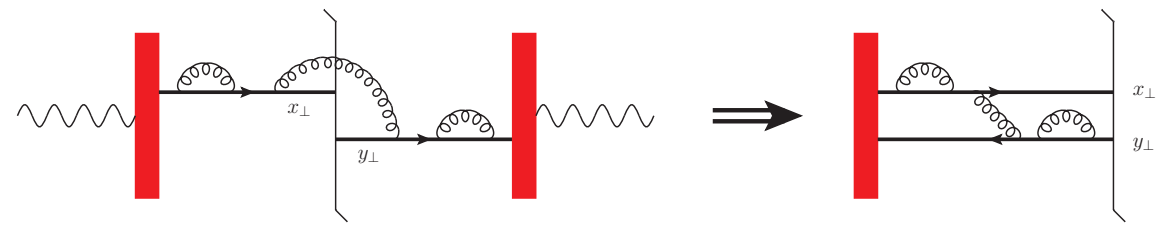

Figure 2: An illustration of the crossing symmetry for the quantum evolution corrections on a pair of semiinfinite Wilson lines. The thick vertical band indicates the nucleus (the shock wave) with all the multiple scatterings on its nucleons.

The quark-quark correlator giving rise to all the TMDs can be written in terms of the Wilson line correlator (1.1),

$$
\begin{aligned}
\Phi^{A}(x, \underline{k} ; P) & =\frac{2 A g_{+-}}{(2 \pi)^{5}} \int d^{2+} p d^{2-} b d^{2} r d^{2} k^{\prime} e^{-i\left(\underline{k}-\underline{k^{\prime}}-\hat{x} \underline{p}\right) \cdot \underline{r}} \\
& \times\left(W_{\text {unp }}(p, b ; P) \phi_{\text {unp }}\left(\hat{x}, \underline{k}^{\prime} ; p\right)-\hat{W}_{p o l, \mu}(p, b ; P) \hat{\phi}_{p o l}^{\mu}\left(\hat{x}, \underline{k}^{\prime} ; p\right)\right) S_{\left(r_{T}, b_{T}\right)}^{\left[\infty^{-}, b^{-}\right]},
\end{aligned}
$$

as was shown in $[1,13]$ and was also presented in M. Sievert's talk (these proceedings). Here

$$
\begin{aligned}
& W_{\text {unp }}(\bar{p}, b)=\frac{1}{2} \sum_{\lambda \lambda^{\prime}}\left[W_{\lambda \lambda^{\prime}}(\bar{p}, b) \mathbf{1}_{\lambda^{\prime} \lambda}\right]=\frac{1}{2} \operatorname{Tr}[W(\bar{p}, b)] \\
& \hat{W}_{p o l}^{\mu}(\bar{p}, b)=\frac{1}{2} \sum_{\lambda \lambda^{\prime}}\left[W_{\lambda \lambda^{\prime}}(\bar{p}, b) \hat{\sigma}_{\lambda^{\prime} \lambda}^{\mu}(\bar{p})\right]=\frac{1}{2} \operatorname{Tr}\left[W(\bar{p}, b) \hat{\sigma}^{\mu}(\bar{p})\right]
\end{aligned}
$$

with $W(\bar{p}, b)$ the Wigner distribution of nucleons in the nucleus, while $W_{u n p}$ and $\hat{W}_{\text {pol }}^{\mu}$ are its unpolarized and polarized projections respectively. Similarly

$$
\begin{aligned}
& \phi_{\text {unp }}(x, \underline{k})=\frac{1}{2} \sum_{\lambda \lambda^{\prime}}\left[\phi_{\lambda \lambda^{\prime}}(x, \underline{k}) \mathbf{1}_{\lambda^{\prime} \lambda}\right]=\frac{1}{2} \operatorname{Tr}[\phi(x, \underline{k})] \\
& \hat{\phi}_{\text {pol }}^{\mu}(x, \underline{k})=\frac{1}{2} \sum_{\lambda \lambda^{\prime}}\left[\phi_{\lambda \lambda^{\prime}}(x, \underline{k}) \hat{\sigma}_{\lambda^{\prime} \lambda}^{\mu}\right]=\frac{1}{2} \operatorname{Tr}\left[\phi(x, \underline{k}) \hat{\sigma}^{\mu}\right]
\end{aligned}
$$

with $\phi(x, \underline{k})$ the quark-quark correlator in a nucleon. 
The equation (1.3) was originally derived in the quasi-classical case, where to evaluate the correlator (1.1) one resums multiple rescattering diagrams $[14,15,16,17]$. The crossing symmetry in Fig. 2 is valid both for multiple rescatterings and for gluon emission [18].

It appears that evolution corrections only contribute to $S$ in Eq. (1.3), leaving the rest of the expression untouched (at large $x$ ). To resum the evolution corrections we only need to include virtual contributions to the semi-infinite dipole evolution. This is straightforward to do and yields the following evolution equation:

$$
Q^{2} \frac{\partial}{\partial Q^{2}} S_{x y}\left[\infty^{-}, b^{-}\right]\left(Q^{2}\right)=-\frac{\alpha_{s} C_{F}}{\pi} \ln \left[Q^{2}(\underline{x}-\underline{y})^{2}\right] S_{x y}\left[\infty^{-}, b^{-}\right]\left(Q^{2}\right) .
$$

The solution of Eq. (1.6) reads

$$
S_{x y}\left[\infty^{-}, b^{-}\right]\left(Q^{2}\right)=\exp \left(-\int_{Q_{0}^{2}}^{Q^{2}} \frac{d \mu^{2}}{\mu^{2}} \frac{\alpha_{s} C_{F}}{\pi} \ln \left[\mu^{2}(\underline{x}-\underline{y})^{2}\right]\right) S_{x y}\left[\infty^{-}, b^{-}\right]\left(Q_{0}^{2}\right)
$$

with the initial conditions $S_{x y}\left[\infty^{-}, b^{-}\right]\left(Q_{0}^{2}\right)$ at $Q_{0}^{2}=\frac{1}{(\underline{x}-\underline{y})^{2}}$ given by [14]

$$
S_{x y}\left[\infty^{-}, b^{-}\right]=\exp \left[-\frac{1}{4}|x-y|_{T}^{2} Q_{s}^{2}\left(\left|\frac{x+y}{2}\right|_{T}\right)\left(\frac{R^{-}\left(\left|\frac{x+y}{2}\right|_{T}\right)-b^{-}}{2 R^{-}\left(\left|\frac{x+y}{2}\right|_{T}\right)}\right) \ln \frac{1}{|x-y|_{T} \Lambda}\right] .
$$

Here $Q_{s}\left(b_{T}\right)$ is the saturation scale at impact parameter $b_{T}, R^{-}\left(b_{T}\right)$ is the longitudinal radius of the nucleus at impact parameter $b_{T}$ and $\left(\frac{R^{-}\left(b_{T}\right)-b^{-}}{2 R^{-}\left(b_{T}\right)}\right)$ is the fraction of nucleons which participate in the final-state rescattering. The logarithm with infrared (IR) regulator $\Lambda$, which is often neglected, is only important for recovering the perturbative large- $k_{T}$ (small- $\left.|x-y|_{T}\right)$ asymptotics.

The exponent in Eq. (1.7) is the well-known Sudakov form-factor [19, 20], previously derived in the saturation literature in $[21,22,23,24]$. We have thus reproduced the leading-order Collins-Soper-Sterman (CSS) evolution equation [20] which is commonly employed for calculating TMDs. For gluon TMDs, where the Wilson lines are adjoint, one would have to replace $C_{F} \rightarrow N_{c}$ in Eq. (1.7). While Eq. (1.7) is written for a fixed coupling constant $\alpha_{s}$, running coupling corrections to it can be included following [25, 26, 27].

\section{Small- $x$ Evolution}

\subsection{Evolution of Unpolarized-Target TMDs}

Now let us consider TMD evolution in the small- $x$ regime, $s \gg Q^{2} \gg \perp^{2}$. This time the true Bjorken- $x$ is small, $x=Q^{2} /\left(s+Q^{2}\right) \ll 1$, and small- $x$ evolution methods are naturally justified. We begin with the unpolarized proton or nucleus TMDs, concentrating on the unpolarized quark TMD $f_{1}^{A}$ first. As usual, the dominant contribution at small- $x$ comes from diagrams which are order- $\alpha_{s}$ suppressed compared to the channel shown in Fig. 1 above. Concentrating on the SIDIS process again, the dominant small- $x$ contribution is pictured in Fig. 3. Comparing Fig. 3 to the lowestorder (no multiple rescatterings) part of Fig. 1, one immediately sees that the former is order- $\alpha_{s}$ suppressed: this is why the channel in Fig. 3 was not considered above. However, at small- $x$ the channel in Fig. 3 is dominant, being enhanced by a power of $1 / x$ compared to the channel in Fig. 1. 


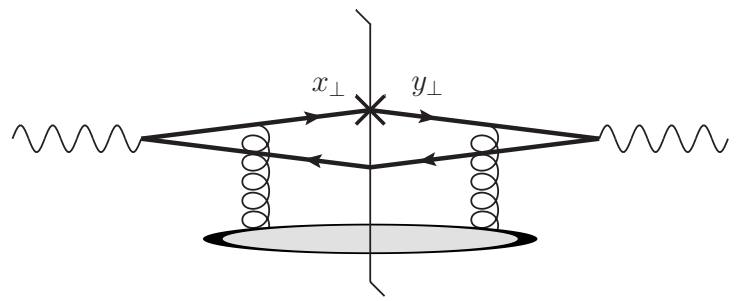

Figure 3: A leading-order contribution to the unpolarized-target SIDIS process at small- $x$. The tagged quark is marked by a cross.

One may ask why the diagram in Fig. 3 was not included along with the evolution corrections considered in Sec. 1. Indeed the quark loop in Fig. 3 generates a factor of $\ln \left(Q^{2} / k_{T}^{2}\right)$, such that the suppression of Fig. 3 compared to the lowest-order part of Fig. 1 is diminished to a factor of $\alpha_{s} \ln \left(Q^{2} / k_{T}^{2}\right)$. However it is well-known that the quark loop cannot generate a logarithm of energy; hence the diagram in Fig. 3 is a single-logarithmic correction, which is beyond the DLA accuracy of the evolution in Eq. (1.7) and can be neglected at large- $x$.

The splitting of a virtual photon into a $q \bar{q}$ pair in Fig. 3 happens typically long before the interaction with the target nucleus, denoted by a shaded oval in Fig. 3. Moreover, in the unpolarized SIDIS case all interaction with the target is eikonal and, hence, spin-independent. No interaction with one given nucleon is a special "knockout" interaction, where spin-dependence could be transferred from the target to the probe, as in [13]. Therefore the unpolarized SIDIS cross section at small- $x$, and the corresponding TMDs, do not have the $b^{-}$dependent effects akin to those at large- $x$ as shown in Eq. (1.3) leading to TMD mixing observed in $[1,13]$ following from Eq. (1.3). We also do not need to worry too much about the details of nucleon dynamics encoded in the nuclear Wigner distribution.
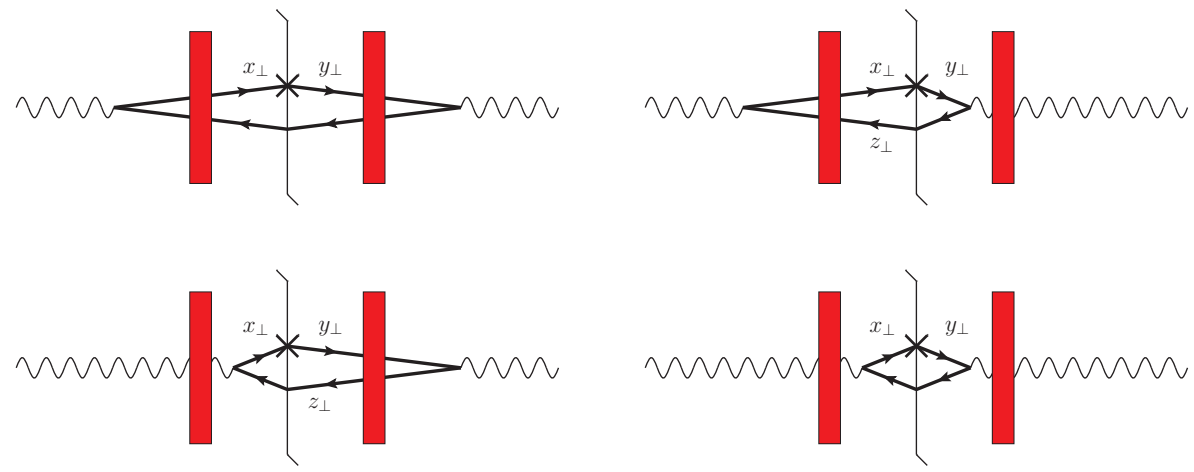

Figure 4: Diagrams representing the sum of all-order contributions to the unpolarized-target SIDIS process at small- $x$. The tagged quark is marked by a cross, the thick vertical band represents the target nucleus (the shock wave).

With this in mind, summing up the diagrams in Fig. 4, where the thick vertical band represents the shock wave and we explicitly show the four contributions where the $\gamma^{*} \rightarrow q \bar{q}$ splitting happens either before or after the photon passes through the shock wave on either side of the cut, we use the light-cone perturbation theory [28] to write the small- $x$ unpolarized quark TMD for a large nucleus 
as [1] (see [29] for a similar result)

$$
\begin{aligned}
& f_{1}^{A}\left(x, k_{T}\right)=\frac{2 N_{c}}{\pi^{3} x} \int \frac{d^{2} x_{\perp} d^{2} y_{\perp} d^{2} z_{\perp}}{2(2 \pi)^{3}} e^{-i \underline{k} \cdot(\underline{x}-\underline{y})} \frac{\underline{x}-\underline{z}}{|\underline{x}-\underline{z}|^{2}} \cdot \frac{\underline{y}-\underline{z}}{|\underline{y}-\underline{z}|^{2}} \\
& \times \frac{|\underline{x}-\underline{z}|^{4}-|\underline{y}-\underline{z}|^{4}-2|\underline{x}-\underline{z}|^{2}|\underline{y}-\underline{z}|^{2} \ln \frac{|\underline{x}-\underline{z}|^{2}}{\mid \underline{y}-\underline{\left.\right|^{2}}}\left[S_{x, y}^{[+\infty,-\infty]}-S_{x, z}^{[+\infty,-\infty]}-S_{z, y}^{[+\infty,-\infty]}+1\right] .}{\left(|\underline{x}-\underline{z}|^{2}-|\underline{y}-\underline{z}|^{2}\right)^{3}}
\end{aligned}
$$

Having constructed the expression (2.1) for the unpolarized quark TMD we can now determine its evolution at small- $x$. It is driven by the small- $x$ evolution of the dipole $S$-matrix. The small- $x$ evolution of $S$ in the leading-logarithmic approximation (LLA) resumming powers of $\alpha_{s} \ln (1 / x)$ is well known and is given by $[5,6,9,10,11,12,30,31]$

$$
\partial_{Y}\left\langle\hat{S}_{x, y}^{[+\infty,-\infty]}\right\rangle_{Y}=\frac{\alpha_{S} N_{c}}{2 \pi^{2}} \int d^{2} z_{\perp} \frac{(\underline{x}-\underline{y})^{2}}{(\underline{x}-\underline{z})^{2}(\underline{z}-\underline{y})^{2}}\left[\left\langle\hat{S}_{x, z}^{[+\infty,-\infty]} \hat{S}_{z, y}^{[+\infty,-\infty]}\right\rangle_{Y}-\left\langle\hat{S}_{x, y}^{[+\infty,-\infty]}\right\rangle_{Y}\right]
$$

with $Y=\ln 1 / x \approx \ln \left(s / Q^{2}\right)$. In Eq. (2.2) we have separated $S_{x y}(Y)=\langle\hat{S}\rangle$ into the Wilson-line operator $\hat{S}$ and the averaging in the nuclear wave function evolved up to rapidity $Y$ (see Eq. (1.1)). Unfortunately Eq. (2.2) is not a closed integro-differential equation: the object $\langle\hat{S} \hat{S}\rangle$ on its righthand side is different from $\langle\hat{S}\rangle$ on the left-hand side. The equation closes in the large- $N_{c}$ limit, where it becomes $[5,6,7,8]$

$$
\partial_{Y} S_{x, y}^{[+\infty,-\infty]}=\frac{\alpha_{S} N_{c}}{2 \pi^{2}} \int d^{2} z_{\perp} \frac{(\underline{x}-\underline{y})^{2}}{(\underline{x}-\underline{z})^{2}(\underline{z}-\underline{y})^{2}}\left[S_{x, z}^{[+\infty,-\infty]} S_{z, y}^{[+\infty,-\infty]}-S_{x, y}^{[+\infty,-\infty]}\right],
$$

where we again dropped the angle brackets. The initial condition for Eq. (2.3) is given by (cf. Eq. (1.8))

$$
S_{x y}^{[+\infty,-\infty]}=\exp \left[-\frac{1}{4}|x-y|_{T}^{2} Q_{s}^{2} \ln \frac{1}{|x-y|_{T} \Lambda}\right] .
$$

Eqs. (2.1), (2.2) and (2.3) give us the expression for the unpolarized quark TMD $f_{1}^{A}$ along with its small- $x$ evolution.

\subsection{Evolution of Polarized Target TMDs: an Outline}

The evolution of polarized target TMDs at small- $x$ is somewhat different from the unpolarized case. Here we will only give the general outline of this evolution, with a more detailed description left for future work [32].

Let us concentrate on the quark TMDs. Again the diagrams of the type shown in Fig. 3 need to be considered, except now one of the quark-gluon vertices is not eikonal, allowing for spin-dependence to be transferred from the target nucleus to the produced quark. Note that, as a consequence, the contribution of the diagram has a factor of $x$ suppression compared to its eikonal contribution to the unpolarized TMDs. These diagrams are supplemented by the same-order (both in $\alpha_{s}$ and in $x$ ) graphs like that shown in Fig. 5.

To include the small- $x$ evolution correction into a generic polarized-target quark TMD one has to start by including the effects of GGM/MV multiple rescatterings [14, 15, 16, 17]. The relevant diagrams are shown in Fig. 6. There the red bar represents the shock wave again. Note that we are 


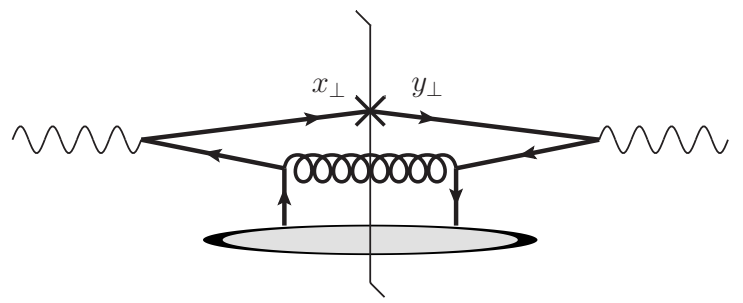

Figure 5: An example of a leading-order contribution to the polarized-target SIDIS process at small- $x$.

treating the rescattering on a nucleon carrying the spin information about the target separately, and draw it explicitly in the graphs. (The spin-dependent scattering may also contain gluon exchanges in the $t$-channel.) Placing such spin-dependent rescattering inside the shock wave rectangle, as shown in Fig. 6, implies that multiple rescatterings may happen both before and after the spindependent scattering.

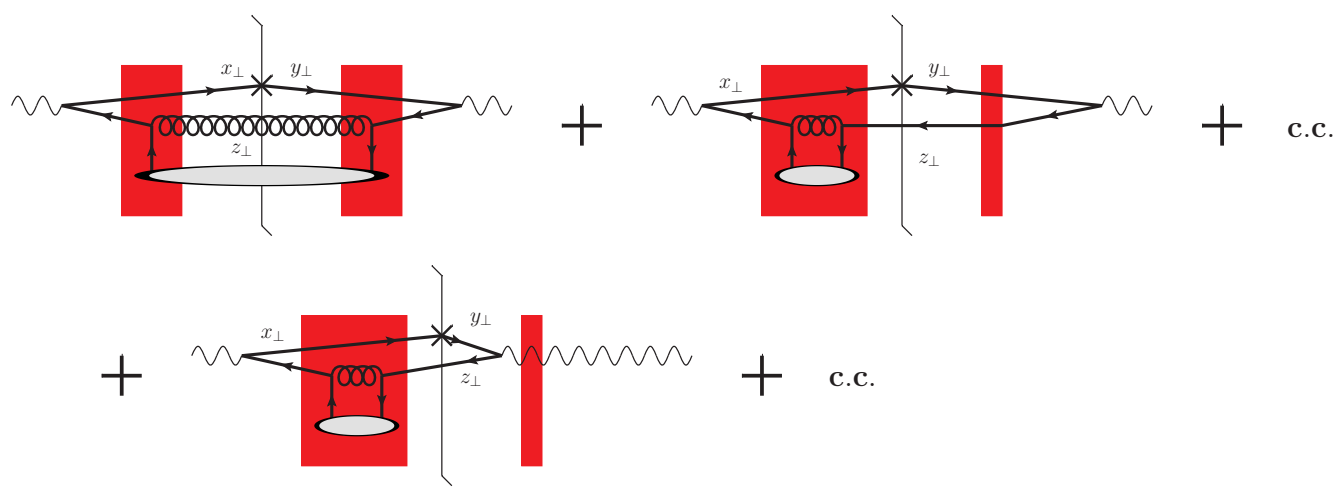

Figure 6: Diagrams needed for the calculation of the polarized SIDIS cross section and the corresponding TMDs.

The spin-dependent scattering preserves the $z_{\perp}$ coordinate of the anti-quark Wilson line. Hence all the scatterings of anti-quark and gluon lines at $z_{\perp}$ cancel between the diagrams in the first line of Fig. 6. This is similar to the reason there is no $z_{\perp}$-dependence in the $S_{x, y}$ representing the interaction with the target in the first graph of Fig. 4. However, in this case we are interested in the target polarization-dependent TMD, and cancellation of the spin-dependent scattering implies that the TMD does not get any contribution from the top line of diagrams in Fig. 6. We are left only with the two diagrams in the second line of Fig. 6. (Note that the photon does not interact with the shock wave it crosses: the shock wave to the right of the cut in the first diagram of the second line of Fig. 6 only indicates that the $\gamma^{*} \rightarrow q \bar{q}$ splitting occurs at positive light-cone time.)

The same arguments apply to small- $x$ evolution corrections to the diagram in Fig. 5 for the polarized TMD at hand. In the end one is left with the diagrams where all the evolution and interaction with the target happen entirely either to the left or to the right of the cut, as illustrated in Fig. 7. For simplicity we only show the linear evolution, realized through the quark ladder exchange shown in the left panel of Fig. 7, and the non-eikonal (non-BFKL) gluon ladder exchange shown in the right panel. The nonlinear evolution corrections are represented by interaction with the shock wave. 

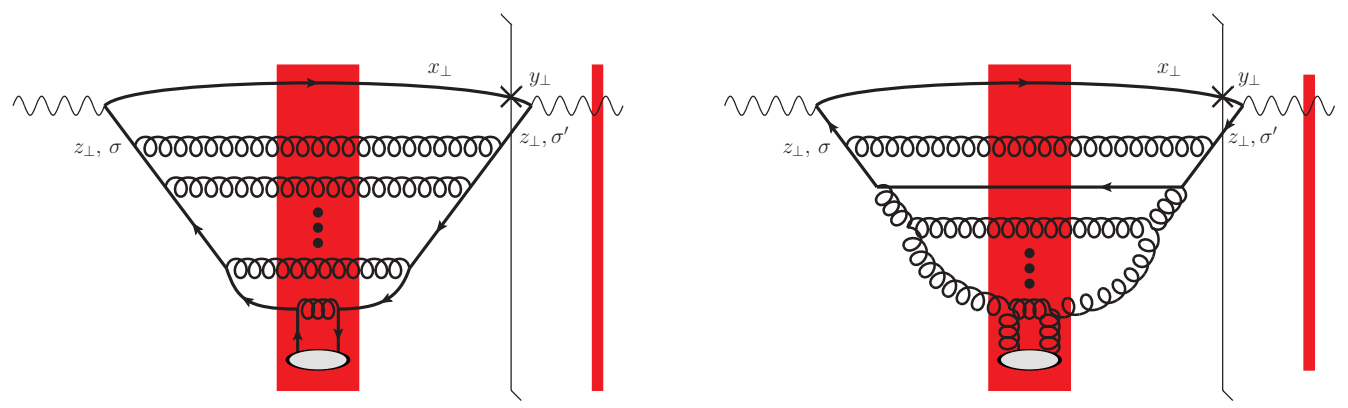

Figure 7: Evolution corrections to the polarized-target SIDIS process at small- $x$. The gluon ladder in the right panel is of the non-BFKL type [33, 34]. The red bands represent the shock wave.

In the left panel of Fig. 7 the anti-quark in the amplitude emits hard gluons, while itself cascading down to lower and lower $x$ until it interacts with the target. After the interaction with the target all the emitted gluons recombine back with the anti-quark. The same applies for the gluon ladder depicted in the right panel of Fig. 7. Combining each diagram with its complex conjugate we write a general polarized-target quark TMD as

$$
\begin{aligned}
f_{A}\left(x, k_{T} ; \lambda, \Sigma\right)=-\int \frac{d^{2} x_{\perp} d^{2} y_{\perp} d^{2} z_{\perp}}{2(2 \pi)^{3}} e^{-i \underline{k} \cdot(\underline{x}-\underline{y})} & \sum_{\sigma, \sigma^{\prime}} \\
& f_{\sigma \sigma^{\prime}}(\underline{x}-\underline{z}, \underline{y}-\underline{z} ; \lambda) \\
\times & {\left[R_{x, z}^{\sigma \sigma^{\prime}}(Y)+R_{y, z}^{\sigma \sigma^{\prime} *}(Y)\right], }
\end{aligned}
$$

where $\lambda$ and $\Sigma$ are the quark and the target spin projections correspondingly (taken in either longitudinal or transverse basis) and $Y \approx \ln 1 / x$ at small- $x$.

Eq. (2.5) contains two main ingredients. The function $f_{\sigma \sigma^{\prime}}(\underline{x}-\underline{z}, \underline{y}-\underline{z} ; \lambda)$ results from the $\gamma^{*} \rightarrow q \bar{q}$ splitting and has to be separately calculated for every polarized TMD by analogy to what was done in the unpolarized target case. The interaction of the $x, z$ (and $y, z$ ) dipole with the target, along with its evolution shown explicitly in Fig. 7, is included through the Reggeon exchange amplitude $R_{x, z}^{\sigma \sigma^{\prime}}(Y)\left(R_{y, z}^{\sigma \sigma^{\prime} *}(Y)\right)$. The evolution of $R_{x, z}^{\sigma \sigma^{\prime}}(Y)$ involves mixing between the quark and gluon ladders, as shown in the right panel of Fig. 7. It is possible that the evolution of the (non-BFKL) gluon ladder depends on the TMD in question. For example, it is conceivable that the evolution of the quark transversity could be different from the quark helicity. While further investigation of this evolution for various polarized TMDs is left for future work, it is likely that, at the leading order, such evolution would consist in resumming double logarithmic corrections, i.e., powers of $\alpha_{s} \ln ^{2}(1 / x)$, similar to the evolution constructed in [35, 36, 37, 38, 33, 34, 39, 40] for various polarized and/or flavor non-singlet observables.

\section{Acknowledgments}

This material is based upon work supported by the U.S. Department of Energy, Office of Science, Office of Nuclear Physics under Award Number DE-SC0004286. MS is supported under DOE Contract No. DE-SC0012704. 


\section{References}

[1] Y. V. Kovchegov and M. D. Sievert, Calculating TMDs of an Unpolarized Target: Quasi-Classical Approximation and Quantum Evolution, arXiv:1505.0117.

[2] A. H. Mueller, Soft gluons in the infinite momentum wave function and the BFKL pomeron, Nucl. Phys. B415 (1994) 373-385.

[3] A. H. Mueller and B. Patel, Single and double BFKL pomeron exchange and a dipole picture of high-energy hard processes, Nucl. Phys. B425 (1994) 471-488, [hep-ph/ 9403256 ].

[4] A. H. Mueller, Unitarity and the BFKL pomeron, Nucl. Phys. B437 (1995) 107-126, [hep-ph/9408245].

[5] I. Balitsky, Operator expansion for high-energy scattering, Nucl. Phys. B463 (1996) 99-160, [hep-ph/9509348].

[6] I. Balitsky, Factorization and high-energy effective action, Phys. Rev. D60 (1999) 014020, [hep-ph/9812311].

[7] Y. V. Kovchegov, Small-x $F_{2}$ structure function of a nucleus including multiple pomeron exchanges, Phys. Rev. D60 (1999) 034008, [hep-ph/9901281].

[8] Y. V. Kovchegov, Unitarization of the BFKL pomeron on a nucleus, Phys. Rev. D61 (2000) 074018, [hep-ph/9905214].

[9] J. Jalilian-Marian, A. Kovner, and H. Weigert, The Wilson renormalization group for low x physics: Gluon evolution at finite parton density, Phys. Rev. D59 (1998) 014015, [hep-ph/970 9432 ].

[10] J. Jalilian-Marian, A. Kovner, A. Leonidov, and H. Weigert, The Wilson renormalization group for low x physics: Towards the high density regime, Phys. Rev. D59 (1998) 014014, [hep-ph/ 9706377 ].

[11] E. Iancu, A. Leonidov, and L. D. McLerran, The renormalization group equation for the color glass condensate, Phys. Lett. B510 (2001) 133-144.

[12] E. Iancu, A. Leonidov, and L. D. McLerran, Nonlinear gluon evolution in the color glass condensate. I, Nucl. Phys. A692 (2001) 583-645, [hep-ph/ 0011241$].$

[13] Y. V. Kovchegov and M. D. Sievert, Sivers Function in the Quasi-Classical Approximation, Phys.Rev. D89 (2014) 054035, [arXiv: 1310.5028$]$.

[14] A. H. Mueller, Small x Behavior and Parton Saturation: A QCD Model, Nucl. Phys. B335 (1990) 115.

[15] L. D. McLerran and R. Venugopalan, Gluon distribution functions for very large nuclei at small transverse momentum, Phys. Rev. D49 (1994) 3352-3355, [hep-ph/9311205].

[16] L. D. McLerran and R. Venugopalan, Green's functions in the color field of a large nucleus, Phys. Rev. D50 (1994) 2225-2233, [hep-ph / 9402335$].$

[17] L. D. McLerran and R. Venugopalan, Computing quark and gluon distribution functions for very large nuclei, Phys. Rev. D49 (1994) 2233-2241, [hep-ph/9309289].

[18] A. Mueller and S. Munier, $p_{\perp}$-broadening and production processes versus dipole/quadrupole amplitudes at next-to-leading order, Nucl.Phys. A893 (2012) 43-86, [arXiv: 1206.1333 ].

[19] V. V. Sudakov, Vertex parts at very high-energies in quantum electrodynamics, Sov. Phys. JETP 3 (1956) 65-71.

[20] J. C. Collins, D. E. Soper, and G. F. Sterman, Factorization of Hard Processes in QCD, Adv.Ser.Direct.High Energy Phys. 5 (1988) 1-91, [hep-ph/ 0409313 ]. 
[21] E. Levin, Gluon saturation and inclusive production at low transverse momenta, Phys.Rev. D82 (2010) 101704, [arXiv:1010.4630].

[22] A. Mueller, B.-W. Xiao, and F. Yuan, Sudakov Resummation in Small-x Saturation Formalism, Phys.Rev.Lett. 110 (2013), no. 8 082301, [arXiv:1210.5792].

[23] A. Mueller, B.-W. Xiao, and F. Yuan, Sudakov double logarithms resummation in hard processes in the small-x saturation formalism, Phys.Rev. D88 (2013), no. 11 114010, [arXiv: 1308 . 2993].

[24] I. Balitsky and A. Tarasov, Evolution of gluon TMD at low and moderate x, Int.J.Mod.Phys.Conf.Ser. 37 (2015) 0058, [arXiv:1411.0714].

[25] I. I. Balitsky, Quark Contribution to the Small-x Evolution of Color Dipole, Phys. Rev. D 75 (2007) 014001, [hep-ph/0609105].

[26] Y. Kovchegov and H. Weigert, Triumvirate of Running Couplings in Small-x Evolution, Nucl. Phys. A 784 (2007) 188-226, [hep-ph/0609090].

[27] E. Gardi, J. Kuokkanen, K. Rummukainen, and H. Weigert, Running coupling and power corrections in nonlinear evolution at the high-energy limit, Nucl. Phys. A784 (2007) 282-340, [hep-ph/0609087].

[28] G. P. Lepage and S. J. Brodsky, Exclusive processes in perturbative quantum chromodynamics, Phys. Rev. D22 (1980) 2157.

[29] A. H. Mueller, Parton saturation at small $x$ and in large nuclei, Nucl. Phys. B558 (1999) 285-303, [hep-ph/9904404].

[30] H. Weigert, Evolution at small $x_{b j}$ : The Color Glass Condensate, Prog. Part. Nucl. Phys. 55 (2005) 461-565, [hep-ph/0501087].

[31] Y. V. Kovchegov, J. Kuokkanen, K. Rummukainen, and H. Weigert, Subleading-N $N_{c}$ corrections in non-linear small-x evolution, Nucl. Phys. A823 (2009) 47-82, [arXiv : 0812 . 3238].

[32] Y. V. Kovchegov, D. Pitonyak, M. D. Sievert, and R. Venugopalan, Small-x evolution of helicity $T M D s$, in preparation.

[33] J. Bartels, B. Ermolaev, and M. Ryskin, Nonsinglet contributions to the structure function g1 at small x, Z.Phys. C70 (1996) 273-280, [hep-ph/9507271].

[34] J. Bartels, B. Ermolaev, and M. Ryskin, Flavor singlet contribution to the structure function $G(1)$ at small x, Z.Phys. C72 (1996) 627-635, [hep-ph/9603204].

[35] R. Kirschner and L. Lipatov, Double Logarithmic Asymptotics and Regge Singularities of Quark Amplitudes with Flavor Exchange, Nucl.Phys. B213 (1983) 122-148.

[36] R. Kirschner, Regge asymptotics of scattering with flavor exchange in QCD, Z.Phys. C67 (1995) 459-466, [hep-th/9404158].

[37] R. Kirschner, Reggeon interactions in perturbative QCD, Z.Phys. C65 (1995) 505-510, [hep-th/9407085].

[38] S. Griffiths and D. Ross, Studying the perturbative Reggeon, Eur.Phys.J. C12 (2000) 277-286, [hep-ph/9906550].

[39] J. Bartels and M. Lublinsky, Quark anti-quark exchange in gamma* gamma* scattering, JHEP 0309 (2003) 076, [hep-ph/0308181].

[40] K. Itakura, Y. V. Kovchegov, L. McLerran, and D. Teaney, Baryon stopping and valence quark distribution at small x, Nucl. Phys. A730 (2004) 160-190, [hep-ph/ 0305332 ]. 